\title{
Presaturation phase with no dipolar order in a quantum ferro-antiferromagnet
}

\author{
V. K. Bhartiya $\odot,{ }^{1,}{ }^{*}$ K. Yu. Povarov, ${ }^{1}$ D. Blosser $\odot,{ }^{1}$ S. Bettler $\odot,{ }^{1}$ Z. Yan $\odot,{ }^{1}$ S. Gvasaliya $\odot,{ }^{1}$ S. Raymond, ${ }^{2}$ \\ E. Ressouche, ${ }^{2}$ K. Beauvois, ${ }^{3}$ J. Xu $\odot,{ }^{4}$ F. Yokaichiya ${ }^{\circ},{ }^{4}$ and A. Zheludev ${ }^{1, \dagger}$ \\ ${ }^{1}$ Laboratory for Solid State Physics, ETH Zürich, 8093 Zürich, Switzerland \\ ${ }^{2}$ Université Grenoble Alpes, CEA, IRIG/DEPHY/MEM-MDN, 38000 Grenoble, France \\ ${ }^{3}$ Institut Laue Langevin, 38000 Grenoble, France \\ ${ }^{4}$ Helmholtz-Zentrum Berlin für Materialien und Energie GmbH, Hahn-Meitner-Platz 1, 14109 Berlin, Germany
}

(Received 5 August 2019; revised manuscript received 11 October 2019; published 6 November 2019)

\begin{abstract}
Magnetization, magnetocaloric, calorimetric, neutron- and x-ray-diffraction, and inelastic-neutron-scattering measurements are performed on single crystals of $\mathrm{BaCdVO}\left(\mathrm{PO}_{4}\right)_{2}$. The low-temperature crystal structure is found to be of a lower symmetry than previously assumed. The result is a more complicated model spin Hamiltonian, which we infer from measurements of the spin wave dispersion spectrum. The main finding is a spin state which emerges in high magnetic fields after antiferromagnetic order is terminated at $H_{c 1} \simeq 4.0 \mathrm{~T}$. It is a distinct thermodynamic phase with a well-defined phase boundary at $H_{c 2} \simeq 6.5 \mathrm{~T}$ and is clearly separate from the fully saturated phase, yet it shows no conventional (dipolar) magnetic long-range order. We argue that it is fully consistent with the expectations for a quantum bond-nematic state.
\end{abstract}

DOI: 10.1103/PhysRevResearch.1.033078

\section{INTRODUCTION}

Conventional long-range order in spin systems is represented by a static, usually periodically modulated magnetization. It breaks both rotational and time-reversal symmetries of the underlying Heisenberg Hamiltonian. Spin-nematic order, as first envisioned by Blume and Hsieh [1] and generalized by Andreev and Grishchuk [2], breaks rotational but not time-reversal symmetries. Spins continue to fluctuate, but these fluctuations spontaneously become anisotropic. From a theorist's perspective, such exotic quantum states are robust and not particularly rare, often realized in partially magnetized systems with competing antiferromagnetic (AF) and ferromagnetic (FM) interactions [3-5]. For example, the simple next-nearest-neighbor (NNN) Heisenberg AF coupling on a square lattice with nearest-neighbor (NN) FM coupling is predicted to always be a spin nematic in some range of applied magnetic fields just below full polarization [6,7]. Finding an experimental realization of even this relatively unconstrained model is a formidable challenge. The only known potential host compounds are layered vanadophosphates of type $A A^{\prime} \mathrm{VO}\left(\mathrm{PO}_{4}\right)_{2}$ [8,9]. Until recently, no unambiguous signatures of spin-nematic phases had been found in any of them. The main obstacle has been a lack of single-crystal samples. Indeed, powder data are notoriously difficult to interpret. In applied magnetic fields, where each crystallite experiences

\footnotetext{
*vvivek@phys.ethz.ch

†zhelud@ethz.ch; http://www.neutron.ethz.ch/
}

Published by the American Physical Society under the terms of the Creative Commons Attribution 4.0 International license. Further distribution of this work must maintain attribution to the author(s) and the published article's title, journal citation, and DOI. a priori different conditions, phase transitions and other anomalies that may be indicative of the nematic state become washed out. Thus, single-crystal experiments are key.

In our recent paper [10] we reported thermodynamic evidence of a presaturation quantum phase in small single crystals of $\mathrm{BaCdVO}\left(\mathrm{PO}_{4}\right)_{2}$, one of the most promising host materials [11]. Powder magnetometric and neutron-diffraction studies by other authors [12] seemingly supported the notion of the phase being the elusive spin-nematic state, but, as argued below, may otherwise be misleading. In the present work we settle this issue decisively. We report combined neutron- and x-ray-diffraction, inelastic neutron-scattering, and Faraday balance magnetometry, calorimetry, and magnetocaloric (MCE) studies on appropriate size $\mathrm{BaCdVO}\left(\mathrm{PO}_{4}\right)_{2}$ single crystals. We first show that the low-temperature crystallographic structure is of a lower symmetry than previously assumed. Consequently, the spin wave spectrum is quite different from that of a simple square lattice. Despite that, the putative presaturation nematic phase exists and persists over a surprisingly wide range of applied magnetic fields. It has distinct thermodynamic phase boundaries, but its polarization is almost complete, over $98 \%$ of full saturation.

\section{MINIMAL MAGNETIC HAMILTONIAN}

At room temperature $\mathrm{BaCdVO}\left(\mathrm{PO}_{4}\right)_{2}$ has an orthorhombic $P_{b c a}\left(D_{2 h}^{15}\right.$, No. 61) structure with eight $\mathrm{V}^{4+}$ ions per unit cell [13] forming an approximate $S=1 / 2$ square lattice in the $(a, b)$ plane. If what is known about the structurally similar $\mathrm{Pb}_{2} \mathrm{VO}\left(\mathrm{PO}_{4}\right)_{2}$ is any guidance [14], magnetic interactions are in these planes, with negligible interlayer coupling. The NN and $\mathrm{NNN}$ in-plane exchange constants for $\mathrm{BaCdVO}\left(\mathrm{PO}_{4}\right)_{2}$ have been previously estimated from powder data as $J_{1}=$ $-0.31 \mathrm{meV}$ and $J_{2}=0.28 \mathrm{meV}$ [15]. There is also a weak 


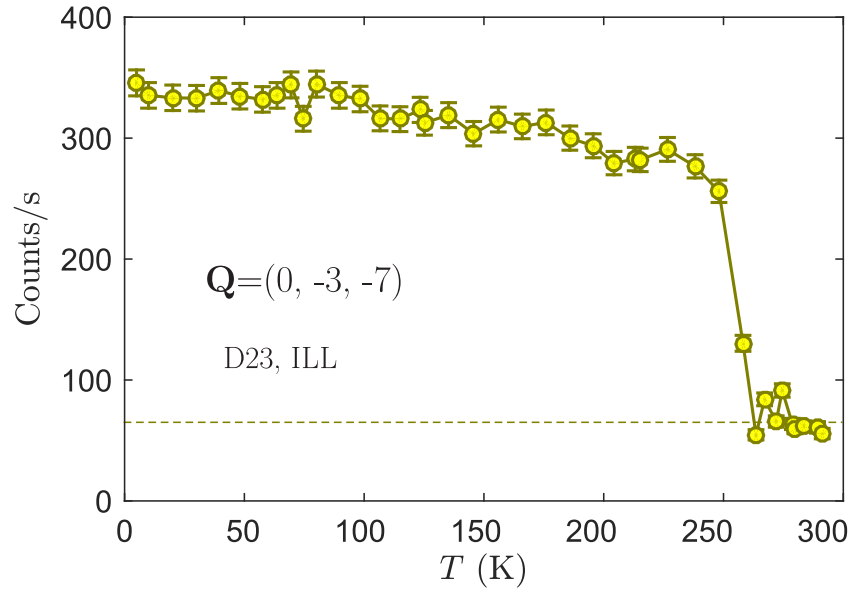

FIG. 1. Temperature dependence of the neutron intensity measured at $(0,-3,-7)$. The corresponding nuclear Bragg reflection is extinct in the $P_{b c a}$ structure. In the data shown a constant background (dashed line) was measured a few degrees off the Bragg position.

easy-axis anisotropy that manifests in a spin flop transition around $\mu_{0} H_{\mathrm{SF}} \simeq 0.5 \mathrm{~T}$ for $\mathbf{H} \| \mathbf{a}$ [10].

Long-range magnetic order in zero applied field is of a peculiar up-up-down-down type, propagation vector $(0,1 / 2,0)$, and appears below $T_{N}=1.05 \mathrm{~K}$ [12]. It is incompatible with the simple $J_{1}-J_{2}$ model and was attributed to additional thirdnearest neighbor interactions in the $\mathrm{V}^{4+}$ layers $[12,16]$.

There is however a more natural explanation. It turns out that $\mathrm{BaCdVO}\left(\mathrm{PO}_{4}\right)_{2}$ undergoes a structural phase transition at $T \sim 250 \mathrm{~K}$. It was detected in a diffraction experiment at the CRG-D23 diffractometer at ILL using 14-meV neutrons. The sample was a 42-mg single crystal. This and all other samples for neutron experiments reported below were grown using $98.8 \%$ isotopically enriched $\mathrm{Cd}^{114}$ to reduce neutron absorbtion. The structural transition manifests itself in the appearance of the $(0,-3,-7)$ Bragg reflection, which is forbidden in $P_{b c a}$ but becomes visible below the transition point (see Fig. 1). At low temperature the compound remains orthorhombic, with the space group reduced to $P_{c a} 2_{1}$ $\left(C_{2 v}^{5}\right.$, No. 29).

The structure was solved at $T=120 \mathrm{~K}$ from $14519 \mathrm{Bragg}$ intensities collected in a single-crystal $\mathrm{x}$-ray-diffraction experiment using a Bruker APEX-II diffractometer. The refined atomic positions (final $R$ factor $R=0.054$ ) and other crystallographic information are presented in the Supplemental Material [17]. The $P_{c a} 2_{1}$ structure, with two inequivalent $\mathrm{V}^{4+}$ positions, allows for as many as four $\mathrm{NN}$ and four NNN interactions within each $\mathrm{V}^{4+}$ layer as shown in the inset of Fig. 2. In particular, unlike at room temperature, there is a possibility of alternating $\mathrm{NN}$ and $\mathrm{NNN}$ interactions along the crystallographic $b$ axis. This naturally explains the $(0,1 / 2,0)$ magnetic propagation vector without invoking three $\mathrm{NN}$ coupling, which would have to span an unrealistic $8-\AA$ distance.

In order to elucidate the effect of lowered crystal symmetry on the coupling constants in $\mathrm{BaCdVO}\left(\mathrm{PO}_{4}\right)_{2}$ we performed preliminary inelastic-neutron-scattering measurements of the spin wave dispersion in the fully saturated phase. The data were collected at $T=70 \mathrm{mK}$ on the IN12 cold neutron three-

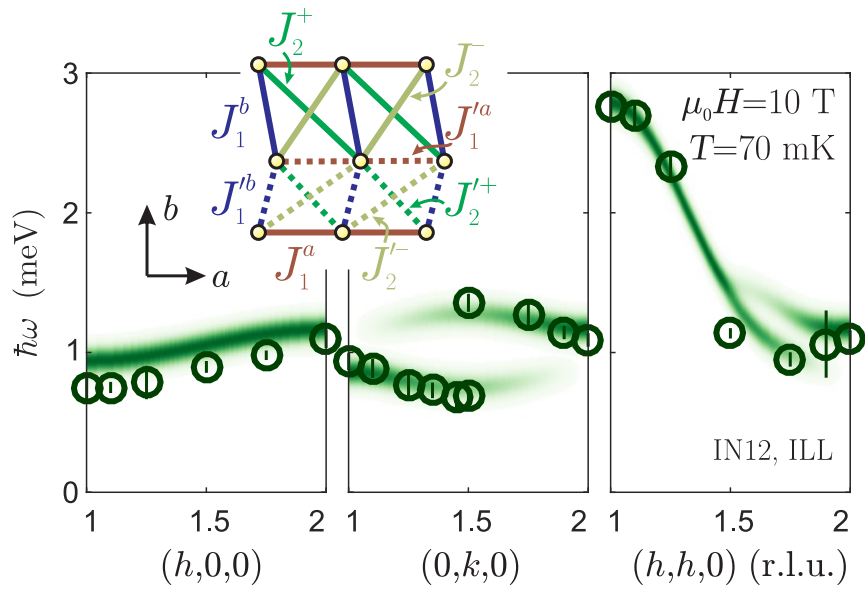

FIG. 2. Spin wave spectrum in fully polarized $\mathrm{BaCdVO}\left(\mathrm{PO}_{4}\right)_{2}$. Symbols are experimental data; the shading in the background shows the intensities from linear spin wave theory. The inset shows the schematic of symmetry-allowed Heisenberg exchange interactions in the $P_{c a} 2_{1}$ structural phase of $\mathrm{BaCdVO}\left(\mathrm{PO}_{4}\right)_{2}$.

axis neutron spectrometer at ILL in a $\mu_{0} H=10 \mathrm{~T}$ magnetic field applied along the crystallographic $c$ axis. We coaligned two $\mathrm{Cd}^{114}$-enriched samples with a total mass of $320 \mathrm{mg}$. The combined sample consisted of five distinct crystallites of roughly equal size, each with a mosaic spread of less than $1^{\circ}$, resulting in an effective mosaic spread of $10^{\circ}$ full width at half height, as estimated from the measured standard deviation of the Bragg intensity distribution in a rocking curve. Typical constant-Q $\mathbf{Q}$ scans performed with $3.5-\mathrm{meV}$ final energy neutrons are shown in Fig. 3. A series of such measurements revealed the dispersion relations plotted in Fig. 2 (symbols). Even though the positions of the observed inelastic peaks are robustly defined, a quantitative analysis of their intensities is not feasible due to large residual neutron absorbtion in the sample that has a rather irregular shape.

The measured dispersion relation was analyzed using linear spin wave theory (SPINW package [18]), which at full saturation becomes exact [19]. The entire set of eight exchange constants cannot be uniquely determined from the three sets of dispersion curves measured in this preliminary experiment. However, we find that without an alternation of $J$ 's along the $b$ direction the two distinct spin wave branches clearly visible in the data cannot be accounted for. With such an alternation, even a restricted minimal model reproduces the measured spectrum reasonably well with $J_{1}^{a}=J_{1^{a}}^{\prime}=J_{1}^{b}=-0.42(1) \mathrm{meV}, J_{1^{b}}^{\prime}=-0.34(1) \mathrm{meV}, J_{2}^{+}=$ $J_{2}^{-}=0.16(1) \mathrm{meV}$, and $J_{2}^{\prime+}=J_{2}^{\prime-}=0.38(1) \mathrm{meV}$. The corresponding calculated scattering intensities [18] are shown as a false color plot in Fig. 2. Further measurements will be needed to uniquely determine the Hamiltonian, but it is already clear that for $\mathrm{BaCdVO}\left(\mathrm{PO}_{4}\right)_{2}$ the simple $J_{1}-J_{2}$ model is inadequate and that an alternation of exchange constants plays a crucial role. An additional outcome of the lowered symmetry could be Dzyaloshinskii-Moriya off-diagonal interactions. However, the highly isotropic low-temperature $g$ factors [10] and the very small spin-flop field suggest that anisotropy effects remain minuscule. 


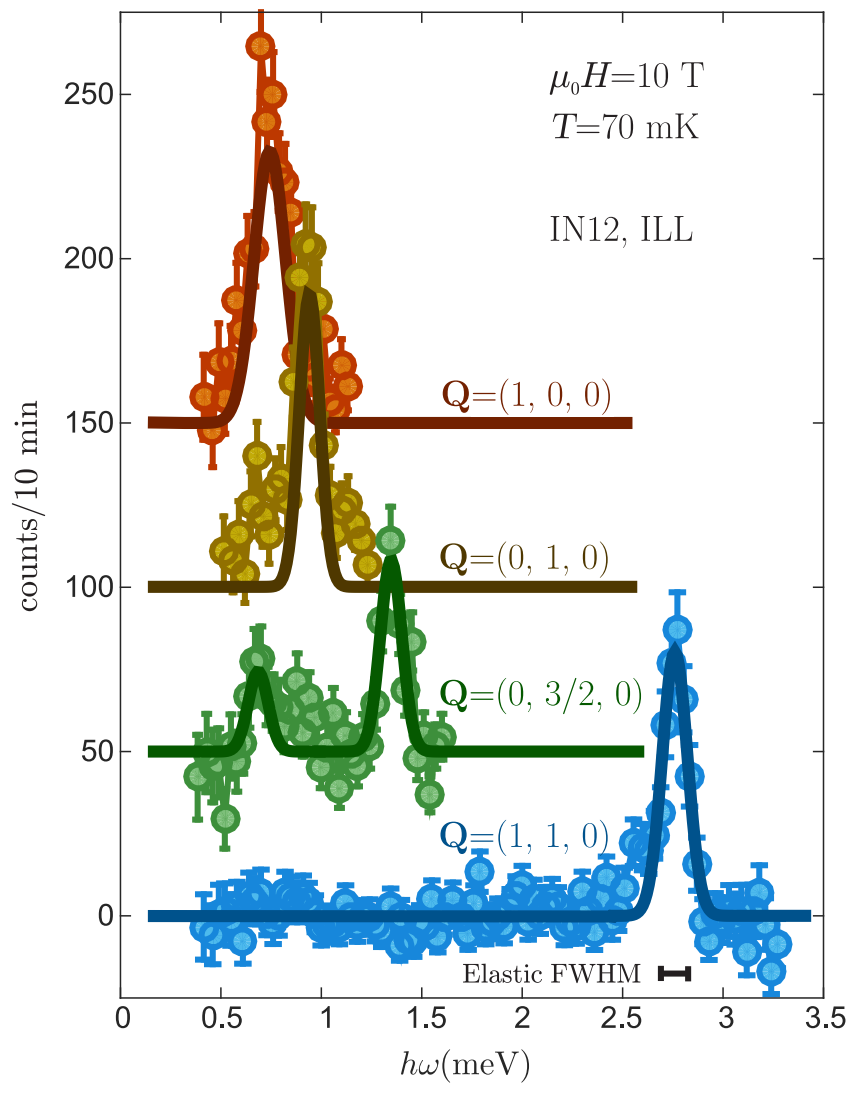

FIG. 3. Representative constant- $\mathbf{Q}$ neutron scans measured in the fully polarized phase $(10 \mathrm{~T} \mathbf{H} \| \mathbf{c})$ in $\mathrm{BaCdVO}\left(\mathrm{PO}_{4}\right)_{2}$ at $70 \mathrm{mK}$. A flat component plus the elastic line was subtracted from all data. An offset of 50 counts/curve was introduced for better visibility. Solid lines are empirical Gaussian fits. For reference, the horizontal bar is the measured energy resolution at elastic position.

\section{PRESATURATION PHASE}

We now turn to the main focus of the present paper, which is the putative low-temperature nematic phase in applied fields. It emerges at $\mu_{0} H_{c 1} \simeq 4 \mathrm{~T}$ in either a firstor a second-order transition for $\mathbf{H} \| \mathbf{a}$ and $\mathbf{H} \| \mathbf{b}$, respectively [10]. The problem with the nematic order parameter is that it is not directly accessible to any experimental technique. ${ }^{1}$ A smoking gun signature can in principle be found in the neutron-excitation spectrum, where the corresponding Goldstone modes vanish near the ordering vector but are visible elsewhere in reciprocal space [11]. Unfortunately, due to the expected small magnitude of the nematic order parameter, these excitations will be at least an order of magnitude weaker than the conventional spin waves. A look at Fig. 3 tells us that observing them in $\mathrm{BaCdVO}\left(\mathrm{PO}_{4}\right)_{2}$ is likely infeasible. The only viable approach to identifying the nematic phase in this material, it is thus a combination of complementary measurements. Figures 4 and 5 show such data for two

\footnotetext{
${ }^{1}$ In principle, it can be probed with resonant magnetic x-ray diffraction. Unfortunately, this technique is incompatible with the high magnetic fields and $\mathrm{mK}$ temperatures at which the nematic state occurs in $\mathrm{BaCdVO}\left(\mathrm{PO}_{4}\right)_{2}$.
}

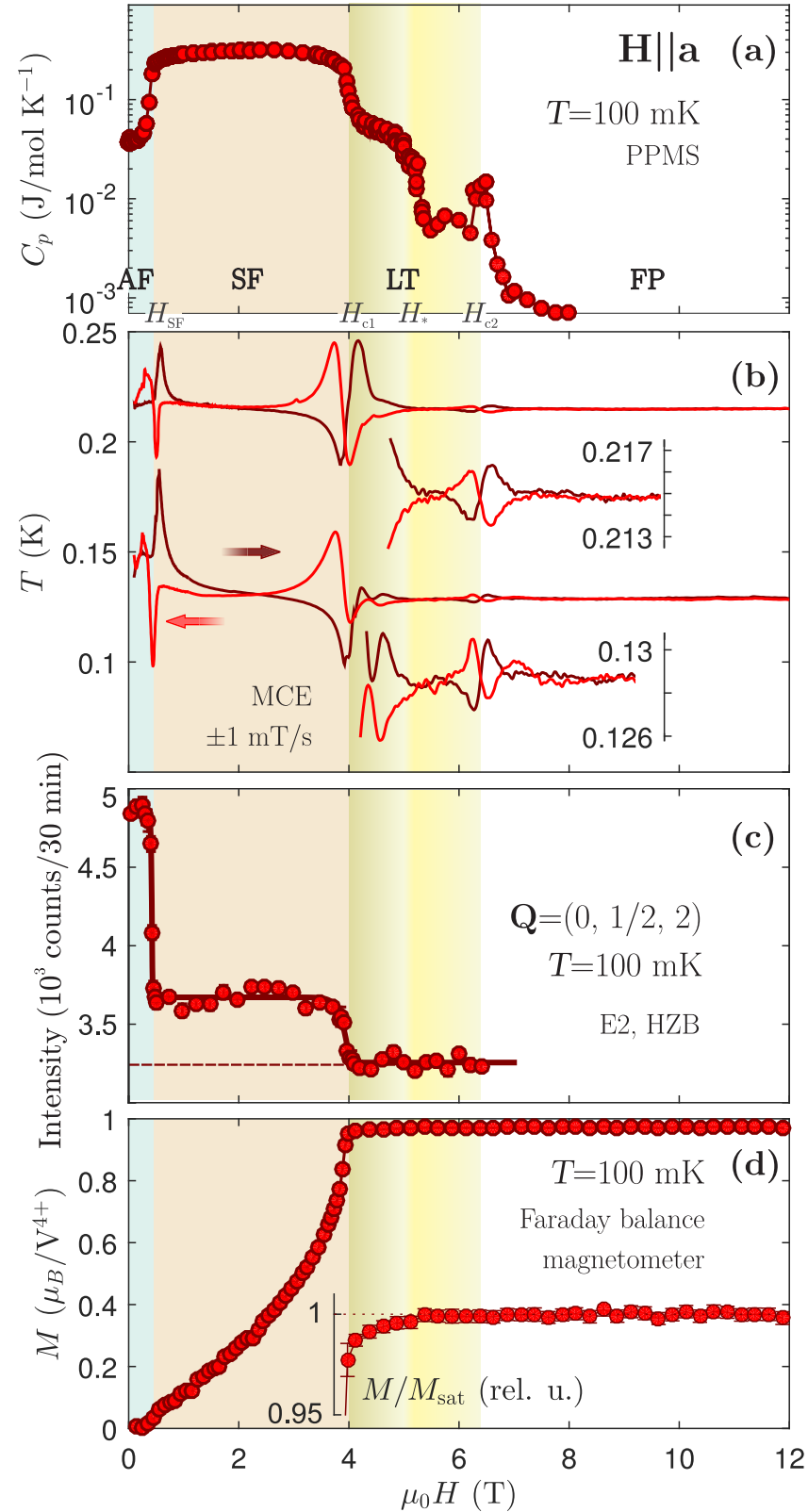

FIG. 4. Magnetic thermodynamics of $\mathrm{BaCdVO}\left(\mathrm{PO}_{4}\right)_{2}$ single crystals in the axial $\mathbf{H} \| \mathbf{a}$ geometry. (a) Specific heat at $100 \mathrm{mK}$. (b) Magnetocaloric effect measurements at 130 and $215 \mathrm{mK}$. The insets shows the fine structure of $T(H)$ curves above $H_{c 1}$ in more detail. (c) Neutron diffraction intensity at the $(0,1 / 2,2)$ magnetic Bragg peak position at $100 \mathrm{mK}$. The solid line is a guide to the eye; the dashed line shows the background intensity. (d) Magnetization curve at $100 \mathrm{mK}$. The inset shows a zoomed in structure of the $M / M_{\text {sat }}$ curve above $H_{c 1}$.

sample geometries, with the magnetic field applied parallel and perpendicular to the magnetic easy axis, respectively. All these measurements are carried out at base temperatures of ${ }^{3} \mathrm{He}-{ }^{4} \mathrm{He}$ dilution refrigerators (100 $\mathrm{mK}$ or lower) on single crystals grown as described in Ref. [10].

The first exhibit [Figs. 4(a) and 5(a)] is heat capacity measured vs applied magnetic field using a commercial Quantum Design PPMS relaxation calorimetry setup on a $2.3-\mathrm{mg}$ 


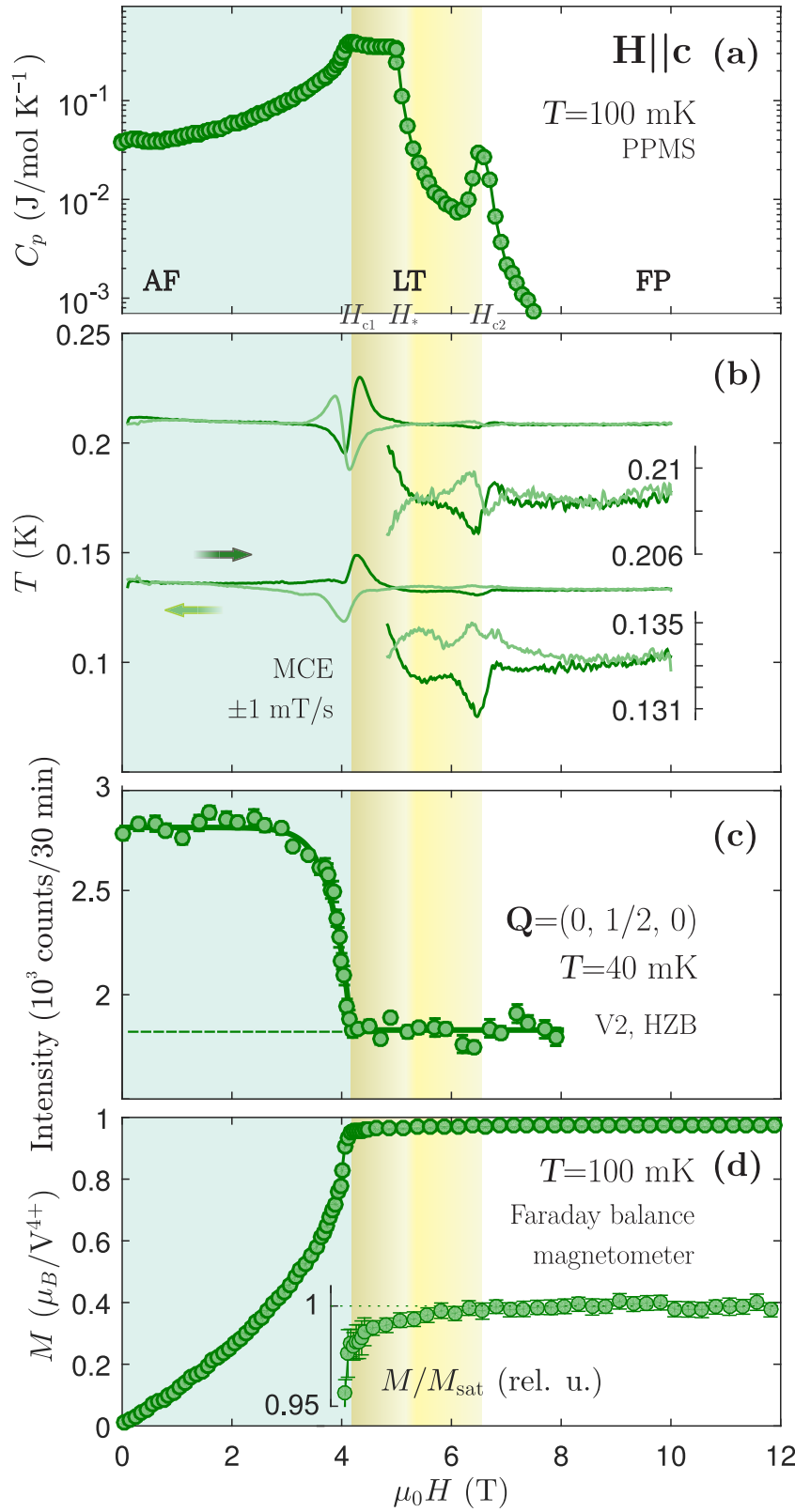

FIG. 5. Measurements similar to those in Fig. 4 in the transverse $\mathbf{H} \| \mathbf{c}$ geometry. (c) Field dependence of the $(0,1 / 2,0)$ magnetic Bragg peak at $40 \mathrm{mK}$.

crystal of $\mathrm{BaCdVO}\left(\mathrm{PO}_{4}\right)_{2}$. As described in detail in Ref. [10], for $\mathbf{H} \| \mathbf{a}$, the discontinuous spin flop transition at $\mu_{0} H_{\mathrm{SF}}$ and another one at $\mu_{0} H_{c 1}=4.00(5) \mathrm{T}$ are marked by sharp steps in the heat capacity. For $\mathbf{H} \| \mathbf{c}$ there is no spin flop transition and the specific heat exhibits a strong divergence approaching $\mu_{0} H_{c 1}=4.08(5) \mathrm{T}$. In both cases, fluctuations persist above $\mu_{0} H_{c 1}$, marking the presaturation state. In all the orientations the specific heat plateau above $\mu_{0} H_{c 1}$ visibly collapses around a crossover field $\mu_{0} H_{*} \simeq 5.5 \mathrm{~T}$. This pronounced change in the resulting specific heat stems from an onset of temperature arrest kind of behavior in the relaxation curves. We interpret it as a hint of the phase separation taking place in the sample $[20,21]$. At any orientation at still higher

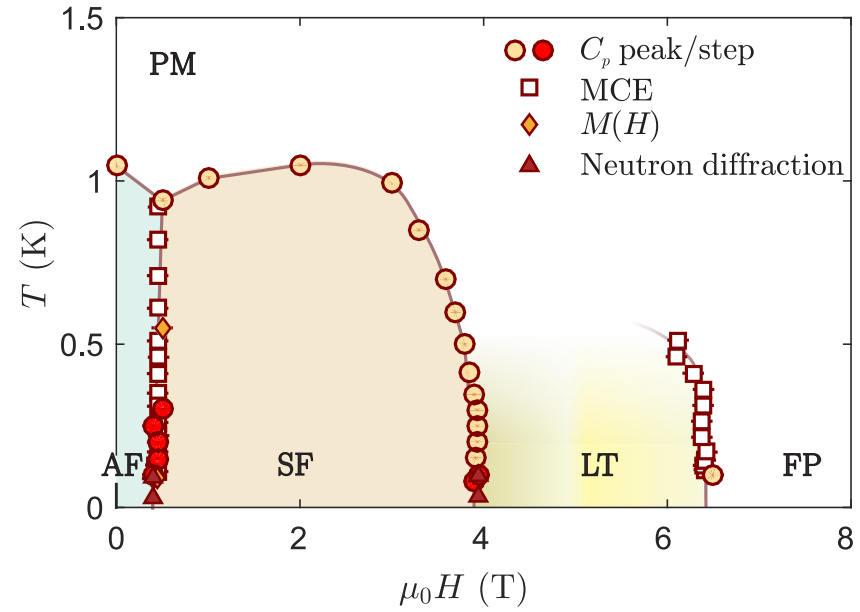

FIG. 6. Magnetic phase diagram of $\mathrm{BaCdVO}\left(\mathrm{PO}_{4}\right)_{2}$ for the $\mathbf{H} \| \mathbf{a}$ field direction obtained by a combination of measurement techniques. Solid lines are a guide to the eye. The phases are as follows: PM and FP, paramagnetic and fully polarized paramagnetic, respectively; AF and SF, conventional antiferromagnetic states before and after spin flop, respectively; and LT, novel presaturation phase presumed to be the quantum spin-nematic state.

field $\mu_{0} H_{c 2} \simeq 6.5 \mathrm{~T}$ there clearly is an additional $\lambda$ anomaly, albeit a weak one. ${ }^{2}$

The transition at $H_{c 2}$ is even more obvious in the second exhibit [Figs. 4(b) and 5(b)]. These are magnetocaloric measurements on the same sample using a constant field sweep rate of $1 \mathrm{mT} / \mathrm{s}$. Up and down sweeps are shown as darker and lighter lines, respectively. For $\mathbf{H} \| \mathbf{a}$ at around $130 \mathrm{mK}$, $H_{\mathrm{SF}}$ and $H_{c 1}$ are both marked by asymmetric anomalies, characteristic of discontinuous transitions [22]. For $\mathbf{H} \| \mathbf{c}$ the lowest-temperature magnetocaloric sweeps, the anomaly at $H_{c 1}$ is also asymmetric. At the same time, a weak but clear magnetocaloric anomaly is also present at $H_{c 2}$ for all the orientations. At slightly elevated temperatures $[T \simeq 210 \mathrm{mK}$ set of curves in Figs. 4(b) and 5(b)] the main anomaly at $H_{c 1}$ turns symmetric, indicative of a continuous phase transition [22]. In both geometries, at slightly elevated temperatures, the transition at $H_{c 2}$ is clearly continuous as well.

Additional magnetocaloric effect scans at different temperatures allow us to trace the $H_{c 2}$ phase boundary vs temperature and reconstruct the $H-T$ phase diagram. The transition can be followed up to about $500 \mathrm{mK}$. Up to $350 \mathrm{mK}$ it barely shifts and shows no sign of broadening; only its magnitude decreases. Above $350 \mathrm{mK}$ the left shoulder of the magnetocaloric anomaly vanishes and it turns into a broad peak. This peak becomes indiscernible in the data noise above $500 \mathrm{mK}$. Figure 6 shows the phase boundaries deduced from MCE studies, magnetization, neutron diffraction, and specific heat [10]. Very similar temperature-dependent behavior was observed for $\mathbf{H} \| \mathbf{c}$ and $\mathbf{H} \| \mathbf{b}$ (not shown). The measured phase diagram for $\mathrm{BaCdVO}\left(\mathrm{PO}_{4}\right)_{2}$ shows certain similarities

\footnotetext{
${ }^{2}$ In both orientations the relaxation curves for $H>H_{*}$ were taken with a pulse length of $\sim 30 \mathrm{~s}$ and interpreted within a simple exponential relaxation (single $\tau$ ) model.
} 
to the one recently reported for volbortite, a very complicated frustrated magnet that is to some extent equivalent to an $S=1 / 2$ model on a frustrated square lattice and also expected to host a spin-nematic phase [23].

Which of the transitions observed in $\mathrm{BaCdVO}\left(\mathrm{PO}_{4}\right)_{2}$ corresponds to a destruction of conventional long-range order? This is clarified by the third exhibit [Figs. 4(c) and 5(c)], which shows the field dependence of the magnetic Bragg peaks $(0,1 / 2,2)$ and $(0,1 / 2,0)$ measured via neutron diffraction. These data were taken on the V2 [Fig. 5(c)] and E2 [Fig. 4(c)] instruments at the BER reactor at HZB using 2.74- and 14.2-meV neutrons and 142- and 24-mg $144 \mathrm{Cd}-$ enriched single crystals, respectively. In both geometries the disappearance of conventional magnetic order exactly coincides with $\mu_{0} H_{c 1}$. That no magnetic structure with a different propagation vector exists in higher fields is clear from powder-diffraction experiments of Ref. [12]. We thus identify the region $H>H_{c 2}$ as fully saturated and the field range $H_{c 1}<H<H_{c 2}$ as corresponding to the potential spinnematic phase.

Of course, this interpretation implies that for $H_{c 1}<H<$ $H_{c 2}$ the system does not achieve full polarization. The fourth exhibit [inset in Figs. 4(d) and 5(d)] puts this supposition to the test. These are direct Faraday force measurements of magnetization at $T=100 \mathrm{mK}$ from a $0.8-\mathrm{mg}$ sample. A small diamagnetic linear contribution was inferred from measurements above $8 \mathrm{~T}$ and subtracted from the data shown. For both geometries, the data show a divergent magnetic susceptibility at $H_{c 1}$, seemingly followed by a complete saturation above. The close-up of the high-field region shown in the insets in Figs. 4 and 5 reveals a different story. In order to reduce noise, the data for $\mu_{0} H>4 \mathrm{~T}$ were binned together in $0.25-\mathrm{T}$ intervals. Between $\mu_{0} H_{c 1}$ and at least $5.5 \mathrm{~T}$ the magnetization visibly lies below the average value for all data above $8 \mathrm{~T}$ (dashed line). The effect is small but clearly outside the measurement uncertainty represented by the error bars.

Incomplete polarization at high field could in principle stem from the small magnetic anisotropy present in the system $[24,25]$. However, this scenario can be excluded. The effect would have to be absent in the axial geometry and in all cases confined to a much narrower field range, comparable to the spin flop field value [25]. The high-field phase is indeed not fully saturated. At the same time its polarization is in all cases over $98 \%$. Can such a highly polarized state support nematic order? Arguably it can, since the latter involves transverse spin components [6,11]. As a reference, consider a classical AF state at $99 \%$ saturation. Despite being almost fully polarized it will still have an angle of as much as $16^{\circ}$ between consecutive spins, sufficient for AF order of transverse spin components. Similarly, such proximity to saturation leaves ample room for nematic correlations in the transverse channel.

\section{CONTRAST WITH PREVIOUS STUDIES}

There are stark discrepancies between our results and the powder measurements of Ref. [12]. In that work the magnetization curves were obtained by integrating AC susceptibility data. The one corresponding to the lowest experimental temperature $T=200 \mathrm{mK}$ is considerably less inflected below $H_{c 1}$ than our curves shown in Figs. 4(d) and 5(d) or even those previously measured at $T=550 \mathrm{mK}$ [10]. The inflection point that the authors take for the analog of $H_{c 1}$ does not correspond to any features in our data for any field geometry. The reported value $\mu_{0} H_{c 1}=3.78 \mathrm{~T}$ is considerably lower than the $\mu_{0} H_{c 1} \gtrsim 4.0 \mathrm{~T}$ observed in single crystals for all geometries. The huge reduction of magnetization in the high-field phase concluded in Ref. [12] is most certainly due to $H_{c 1}$ being underestimated.

The upper boundary assigned to the nematic phase in Ref. [12] does not correspond to any thermodynamic anomalies in single crystals. Counterintuitively, it moves out to higher fields with increased temperature. As is quite obvious from previously published single-crystal data [10], this simply indicates a broadening of the cusp in magnetization due to finite temperature. Our present data instead suggest that the upper phase boundary is more or less parallel to the lower one, as one would expect. We cannot speculate on the origin of these discrepancies, except to note the intrinsic limitations of powder experiments in the applied field and the difficulties of dealing with a dissipative component in AC susceptibility admitted by the authors of Ref. [12].

\section{CONCLUSION}

$\mathrm{BaCdVO}\left(\mathrm{PO}_{4}\right)_{2}$ is not a square lattice model material as it was advertised to be. Instead, it has significantly alternating interactions along the $b$ direction. Nevertheless, it features strong FM-AF frustration, the main ingredient for a presaturation bond-nematic phase. While the corresponding order parameter is fundamentally inaccessible to direct measurements, at the lowest temperatures, in a wide field range between $\mu_{0} H_{c 1} \simeq 4.0 \mathrm{~T}$ and $\mu_{0} H_{c 2} \simeq 6.5 \mathrm{~T}$ we find an almost fully polarized thermodynamic phase. It does not support conventional (dipolar) magnetic order but is fully consistent with our expectations for a quantum bond nematic.

\section{ACKNOWLEDGMENTS}

This work was supported by the Swiss National Science Foundation, Division II. The instrument beamtime at D23 and IN12 was supported by the Swiss State Secretariat for Education, Research and Innovation through a CRG grant. We would like to acknowledge Dr. M. Reehius at HZB for his help with checking the quality of single crystals as well as the sample environment team of the ILL facility for their help with our experiments.
[1] M. Blume and Y. Y. Hsieh, Biquadratic exchange and quadrupolar ordering, J. Appl. Phys. 40, 1249 (1969).

[2] A. F. Andreev and I. A. Grishchuk, Spin nematics, Sov. Phys. JETP 60, 267 (1984).
[3] J. Sudan, A. Lüscher, and A. M. Läuchli, Emergent multipolar spin correlations in a fluctuating spiral: The frustrated ferromagnetic spin- $\frac{1}{2}$ Heisenberg chain in a magnetic field, Phys. Rev. B 80, 140402(R) (2009). 
[4] M. E. Zhitomirsky and H. Tsunetsugu, Magnon pairing in quantum spin nematic, Europhys. Lett. 92, 37001 (2010).

[5] L. Balents and O. A. Starykh, Quantum Lifshitz Field Theory of a Frustrated Ferromagnet, Phys. Rev. Lett. 116, 177201 (2016).

[6] N. Shannon, T. Momoi, and P. Sindzingre, Nematic Order in Square Lattice Frustrated Ferromagnets, Phys. Rev. Lett. 96, 027213 (2006).

[7] H. T. Ueda, Magnetic phase diagram slightly below the saturation field in the stacked $J_{1}-J_{2}$ model in the square lattice with the $J_{C}$ interlayer coupling, J. Phys. Soc. Jpn. 84, 023601 (2015).

[8] A. A. Tsirlin and H. Rosner, Extension of the spin- $\frac{1}{2}$ frustrated square lattice model: The case of layered vanadium phosphates, Phys. Rev. B 79, 214417 (2009).

[9] A. A. Tsirlin, B. Schmidt, Y. Skourski, R. Nath, C. Geibel, and H. Rosner, Exploring the spin- $\frac{1}{2}$ frustrated square lattice model with high-field magnetization studies, Phys. Rev. B 80, 132407 (2009).

[10] K. Y. Povarov, V. K. Bhartiya, Z. Yan, and A. Zheludev, Thermodynamics of a frustrated quantum magnet on a square lattice, Phys. Rev. B 99, 024413 (2019).

[11] A. Smerald, H. T. Ueda, and N. Shannon, Theory of inelastic neutron scattering in a field-induced spin-nematic state, Phys. Rev. B 91, 174402 (2015).

[12] M. Skoulatos, F. Rucker, G. J. Nilsen, A. Bertin, E. Pomjakushina, J. Ollivier, A. Schneidewind, R. Georgii, O. Zaharko, L. Keller, C. Rüegg, C. Pfleiderer, B. Schmidt, N. Shannon, A. Kriele, A. Senyshyn, and A. Smerald, Putative spin-nematic phase in $\mathrm{BaCdVO}\left(\mathrm{PO}_{4}\right)_{2}$, Phys. Rev. B 100, 014405 (2019).

[13] S. Meyer, B. Mertens, and H. Müller-Buschbaum, $\mathrm{SrZnVO}\left(\mathrm{PO}_{4}\right)_{2}$ and $\mathrm{BaCdVO}\left(\mathrm{PO}_{4}\right)_{2}$ : Vanadylphosphates related to but not isotypic with the $\mathrm{BaZnVO}\left(\mathrm{PO}_{4}\right)_{2}$ type, Z. Naturforsch. B 52, 985 (1997).

[14] S. Bettler, F. Landolt, Ö. M. Aksoy, Z. Yan, S. Gvasaliya, Y. Qiu, E. Ressouche, K. Beauvois, S. Raymond, A. N. Ponomaryov, S. A. Zvyagin, and A. Zheludev, Magnetic structure and spin waves in the frustrated ferro-antiferromagnet $\mathrm{Pb}_{2} \mathrm{VO}\left(\mathrm{PO}_{4}\right)_{2}$, Phys. Rev. B 99, 184437 (2019).

[15] R. Nath, A. A. Tsirlin, H. Rosner, and C. Geibel, Magnetic properties of $\mathrm{BaCdVO}\left(\mathrm{PO}_{4}\right)_{2}$ : A strongly frustrated spin- $\frac{1}{2}$ square lattice close to the quantum critical regime, Phys. Rev. B 78, 064422 (2008).

[16] P. Sindzingre, L. Seabra, N. Shannon, and T. Momoi, Phase diagram of the spin-1/2 $J_{1}-J_{2}-J_{3}$ Heisenberg model on the square lattice with ferromagnetic $J_{1}$, J. Phys.: Conf. Ser. 145, 012048 (2009); P. Sindzingre, N. Shannon, and T. Momoi, Phase diagram of the spin-1/2 $J_{1}-J_{2}-J_{3}$ Heisenberg model on the square lattice, ibid. 200, 022058 (2010).

[17] See Supplemental Material at http://link.aps.org/supplemental/ 10.1103/PhysRevResearch.1.033078 for refined atomic positions and other crystallographic information.

[18] S. Toth and B. Lake, Linear spin wave theory for single- $Q$ incommensurate magnetic structures, J. Phys.: Condens. Matter 27, 166002 (2015).

[19] R. Coldea, D. A. Tennant, K. Habicht, P. Smeibidl, C. Wolters, and Z. Tylczynski, Direct Measurement of the Spin Hamiltonian and Observation of Condensation of Magnons in the 2D Frustrated Quantum Magnet $\mathrm{Cs}_{2} \mathrm{CuCl}_{4}$, Phys. Rev. Lett. 88, 137203 (2002).

[20] S. Rößler, D. Cherian, W. Lorenz, M. Doerr, C. Koz, C. Curfs, Y. Prots, U. K. Rößler, U. Schwarz, S. Elizabeth, and S. Wirth, First-order structural transition in the magnetically ordered phase of $\mathrm{Fe}_{1.13} \mathrm{Te}$, Phys. Rev. B 84, 174506 (2011).

[21] H. T. Ueda and T. Momoi, Nematic phase and phase separation near saturation field in frustrated ferromagnets, Phys. Rev. B 87, 144417 (2013).

[22] Y. Kohama, C. Marcenat, T. Klein, and M. Jaime, AC measurement of heat capacity and magnetocaloric effect for pulsed magnetic fields, Rev. Sci. Instrum. 81, 104902 (2010).

[23] Y. Kohama, H. Ishikawa, A. Matsuo, K. Kindo, N. Shannon, and Z. Hiroi, Possible observation of quantum spin-nematic phase in a frustrated magnet, Proc. Natl. Acad. Sci. USA 116, 10686 (2019).

[24] R. Hagemans, J.-S. Caux, and U. Löw, Gapped anisotropic spin chains in a field, Phys. Rev. B 71, 014437 (2005).

[25] A. L. Chernyshev, Effects of an external magnetic field on the gaps and quantum corrections in an ordered Heisenberg antiferromagnet with Dzyaloshinskii-Moriya anisotropy, Phys. Rev. B 72, 174414 (2005). 